\section{(2) OPEN ACCESS}

\title{
Mortality and morbidity in patients with congenital heart disease hospitalised for viral pneumonia
}

\author{
Gerhard-Paul Diller (1) , ${ }^{1,2}$ Dominic Enders, ${ }^{3}$ Astrid E Lammers, ${ }^{1,4}$ Stefan Orwat, ${ }^{1}$ \\ Renate Schmidt, ${ }^{1}$ Robert M Radke 이, ${ }^{1}$ Joachim Gerss, ${ }^{3}$ Fernando De Torres Alba (1) , \\ Gerrit Kaleschke, ${ }^{1}$ Ulrike M Bauer, ${ }^{2}$ Ursula Marschall, ${ }^{5}$ Helmut Baumgartner ${ }^{1}$
}

- Additional material is published online only. To view, please visit the journal online (http://dx.doi.org/10.1136/ heartjnl-2020-317706).

${ }^{1}$ Adult Congenital and Valvular Heart Disease Center, Department of Cardiology and Angiology, University Hospital Muenster, Muenster, Germany ${ }^{2}$ Competence Network for Congenital Heart Defects, Deutsches Herzzentrum Berlin, Berlin, Germany ${ }^{3}$ Institute of Biostatistics and Clinical Research, University of Muenster, Muenster, Germany ${ }^{4}$ Paediatric Cardiology, University of Münster, Münster Germany

${ }^{5}$ Department of Medicine and Health Services Research, BARMER Health Insurance, Wuppertal, Germany

\section{Correspondence to} Professor Gerhard-Paul Diller, Adult Congenital and Valvular Heart Disease Center, Department of Cardiology and Angiology, University Hospital Muenster, Muenster 48149,

Germany;

Gerhard.Diller@ukmuenster.de

Received 1 July 2020

Revised 28 September 2020

Accepted 30 September 2020

Published Online First

27 October 2020

\section{ABSTRACT \\ Objectives Data on the clinical outcome of patients with congenital heart disease (CHD) affected by severe viral pneumonia are limited. We analysed morbidity and mortality of viral pneumonia and evaluated the association between medical conditions, medication, vaccination and outcome specifically in patients with CHD requiring hospitalisation for viral pneumonia.}

Methods Based on data from one of Germany's largest health insurers, all cases of viral pneumonia requiring hospital admission (2005-2018) were studied. Mortality, and composites of death, transplantation, mechanical circulatory support, ventilation or extracorporeal lung support served as endpoints.

Results Overall, 26262 viral pneumonia cases occurred in 24980 patients. Of these, 1180 cases occurred in patients with CHD. Compared with patients without CHD, mortality rate was elevated in patients with CHD. As a group, patients with CHD aged $20-59$ years even exceeded mortality rates in patients without CHD aged $>60$ years. No mortality was observed in patients with CHD with simple defects $<60$ years of age without associated cardiovascular risk factors. On multivariable logistic regression analysis, age, CHD complexity, chromosomal anomalies, cardiac medication, use of immunosuppressants and absence of vaccination for influenza emerged as risk factors of adverse outcome. Conclusions We present timely data on morbidity and mortality of severe viral pneumonia requiring hospital admission in patients with CHD. Need for mechanical ventilation and risk of death in CHD increase early in life, reaching a level equivalent to non-CHD individuals $>60$ years of age. Our data suggest that except for patients with isolated simple defects, patients with CHD should be considered higher-risk individuals when faced with severe viral pneumonia.

\section{INTRODUCTION}

Life trajectories of patients with congenital heart disease (CHD) have been transformed over the past decades. Medical, surgical and catheter interventional progress has enabled most patients with CHD to live longer lives and many now to survive to retirement age. ${ }^{1-3}$ Nonetheless, millions of patients with CHD living in North America and Europe cannot be regarded as cured by these previous reparative measures and frequently present with cardiac and extracardiac complications. ${ }^{245}$ These complications include heart failure, arrhythmias, pulmonary disease, neurological as well as renal and hepatic disease. ${ }^{6}$ Infections such as myocarditis and endocarditis also occur frequently in patients with CHD and are associated with high morbidity and mortality. ${ }^{7}$ Furthermore, over $30 \%$ of adult patients with CHD with cardiac defects of moderate and high complexity have been reported to have at least moderately impaired lung function. ${ }^{8}$

The SARS-CoV-2 pandemic causing COVID-19 has highlighted the potential for severe morbidity and mortality associated with viral disease in various cardiovascular cohorts. Beyond respiratory problems-in particular acute respiratory distress syndrome in adults (ARDS)—cardiovascular complications such as acute myocarditis, heart failure, arrhythmias and heart attacks have been reported. Patients with pre-existing cardiovascular conditions have been found to be more commonly affected, and cardiovascular disease seems to predispose patients to severe or lethal courses of the infection. ${ }^{9}$ Furthermore, increases in creatine kinase, myoglobin and troponin levels have been reported, suggesting virus-induced acute myocardial damage. ${ }^{10}$ Interestingly, the increased fatality rate observed in cardiovascular patients infected with COVID-19 is consistent with data from previous influenza epidemics and similarities between the conditions have been highlighted. ${ }^{11}{ }^{12}$ While unique in the magnitude of its global impact, the currently observed COVID-19 pandemic is, however, not the first of its kind. Since the year 2000, major epidemics of global scale have been recorded, including the SARS epidemic (2002-2004, fatality $>900$ individuals) and the H1N1-influenza-A pandemic (2009, estimated fatality 200000$).{ }^{13}$ In addition, seasonal influenza remains an ongoing issue, having claimed approximately 330000 lives in the USA alone between 2010 and 2019 according to Centre of Disease Control data. ${ }^{14}$

Severe respiratory viral infections are generally accompanied by a sudden increase in lifethreatening cardiac complications in predisposed patients. People with $\mathrm{CHD}$ and their healthcare providers are understandably concerned about the implication of severe viral infections for the CHD population. ${ }^{15}$ Recognising pathophysiological, clinical and radiological similarities between viral pneumonias, ${ }^{11} 16-18$ we used available data to shed light onto the risk of death or need for intensive care management, mechanical ventilation or extracorporeal lung support (ECLS) in this vulnerable
To cite: Diller G-P, Enders $\mathrm{D}$ Lammers AE, et al. Heart 
population afflicted by viral pneumonias. In addition, we evaluated the association between demographics, medical history, history of cardiac and non-cardiac comorbidities as well as medication and vaccination use on outcome.

\section{PATIENTS AND METHODS}

This was a retrospective cohort study based on administrative data from the BARMER GEK German Health Insurance Company. The validity and integrity of the database has been demonstrated and the database has been shown to be representative of the German population. ${ }^{19}$ As data entry is mandatory for administrative purposes, the dataset is complete. The BARMER insures approximately 9 million members (ie, around one out of nine of the German population). All inpatient and outpatient procedures and diagnoses occurring between 2005 and 2018 in the insured population are routinely coded in the BARMER database for reimbursement purposes and were available in an anonymised form for analysis. In addition, information on immunisations against influenza and pneumococcal disease as well as all drug prescriptions (coded according to the Anatomical Therapeutic Chemical Classification System) were also available for analysis. Viral pneumonia was identified based on the relevant inpatient 10 th revision of the International Classification of Diseases (ICD-10) codes as shown in online supplemental appendix, table A. These included specific codes for viral pneumonia (J10-J12) as well as combinations of pneumonia with a concomitant coded detection of influenza viruses. Endpoints of the study included (1) all-cause in-hospital mortality (or death within 30 days of hospital discharge), (2) a combined endpoint of death, successful resuscitation, use of mechanical cardiac support or heart transplantation and (3) use of ECLS, death or resuscitation. Patients with $\mathrm{CHD}$ were identified based on their inpatient or outpatient ICD-10 codes (Q20-Q28 in the German modification of the ICD-10; for details, see online supplemental appendix, table B). Patients were categorised into simple, moderate or severe complexity defect groups based on current guideline recommendation. ${ }^{5}$ Patients with isolated atrial septal defects were not included as this defect shares the same ICD code (Q21.1) with patent foramen ovale in the German modification of ICD-10 codes.

A comprehensive panel of risk factors were included as covariables and are presented in detail in online supplemental appendix, table C. Briefly, we considered pre-existing cardiovascular conditions (such as prior heart failure, myocardial infarction or stroke), extracardiac comorbidities (eg, diabetes, renal or hepatic dysfunction), chronic lung disease, immune disorders, chromosomal anomalies, cancer as well as various cardiac and non-cardiac medications.

\section{Patient and public involvement}

While for this particular project no direct patient involvement was sought, patients and the public are constantly involved in the process of prioritising projects and focusing research on patient-relevant aspects of CHD within the framework of the National Register for Congenital Heart Disease.

\section{Statistical analysis}

Categorical variables are presented as numbers and percentages, while continuous variables are shown as median and IQRs, respectively. Differences between groups are assessed by Mann-Whitney U test or Fisher's exact test depending on data type. To investigate the association between demographics, preexisting medical conditions, immunisations and medication use and outcome, univariable and multivariable logistic regression analyses are presented. The regression models include a random intercept to account for the clustering of the data, that is, individuals being represented more than once in the analysis. The $\mathrm{R}$ statistical software was used for all analyses and a two-sided p-value $<0.05$ was considered significant throughout the study.

\section{RESULTS}

Over the study period, 26262 cases of viral pneumonia requiring hospitalisation occurred in 24980 patients (51.2\% males, median age 33 years (IQR 1-74; age range 0-105 years)). Of these, 1180 cases occurred in 1040 patients with CHD (54.3\% males, median age 2 years (IQR 1-3; age range $0-90$ years), $72.8 \%$ with simple underlying defects, $15.6 \%$ with defects of moderate complexity and $11.6 \%$ with defects of severe complexity. Overall, the age distribution at diagnosis of viral pneumonia followed a bimodal distribution with a peak in early childhood and a second peak after 70 years of age. Across the entire cohort, influenza viruses accounted for $47.1 \%$ of infections, followed by respiratory syncytial viruses (RSVs) with $28.7 \%$, adenoviruses (2.7\%), parainfluenza $(1.8 \%)$ and human metapneumovirus $(1.3 \%)$. In $20.4 \%$ of cases, no specific virus was coded. In the paediatric population ( $<18$ years of age), RSV infection was the most common cause accounting for $55.5 \%$ of cases, followed by influenza (14.2\%), adenoviruses (4.5\%), human metapneumovirus $(1.8 \%)$ and parainfluenza (1.3\%). Interestingly, however, influenza, parainfluenza and human metapneumovirus were relatively over-represented in children who died, required resuscitation, mechanical cardiac support or transplantation $(28.3 \%$ vs $14.2 \%, 5.8 \%$ vs $1.3 \%$ and $5.0 \%$ vs $1.8 \%$, respectively, in the event group vs the overall paediatric population). In contrast, RSV was relatively under-represented in patients with events compared with the entire population (41.7\% vs $55.5 \%)$. In adult patients, influenza accounted for $76.8 \%$ of cases. Further data on the distribution of virus diagnosis between the age groups and specific information about the virus types are provided in the online supplemental material. Table 1 shows baseline parameters stratified by endpoints across the entire study population.

In-hospital case fatality rate (CFR) overall was $8.5 \%$ with important differences between age groups and patients with and without CHD: children with CHD aged 0-9 years had a hospital CFR of $1.3 \%$ compared with $0.3 \%$ in non-CHD children of the same age group. Similarly, CHD children and adolescents aged 10-19 years had a higher mortality rate compared with nonCHD peers (8.5\% vs 3.0\%). Only 40 patients with CHD aged 20-59 years of age required hospitalisation for virus pneumonia during the study period and were thus included in the analysis. However, six of these patients died (hospital CFR 15\%), compared with $9.5 \%$ in patients without CHD of the same age group. As a group, in-hospital CFR in the 20-59-year-old CHD cohort exceeded the CFR seen in patients without CHD aged 60-69 years of age (14.4\%) and approached the mortality rate found in 70-79-year-old patients without CHD (17.2\%) (figure 1).

The composite endpoint of death, successful resuscitation, mechanical cardiac support or heart transplantation occurred in $4.0 \%, 10.6 \%$ and $22.5 \%$ of patients with $\mathrm{CHD}$ and $0.5 \%$, $3.1 \%$ and $13.7 \%$ of patients without CHD aged $0-9,10-19$ and 20-59 years, respectively. For comparison, the event rates in patients without CHD aged 60-69, 70-79 and 80-89 years of age for this endpoint were $17.7 \%, 18.8 \%$ and $21.6 \%$, respectively. Thus, adult CHD had higher event rates compared with those observed in over the 80-year-old non-congenital cohort. 


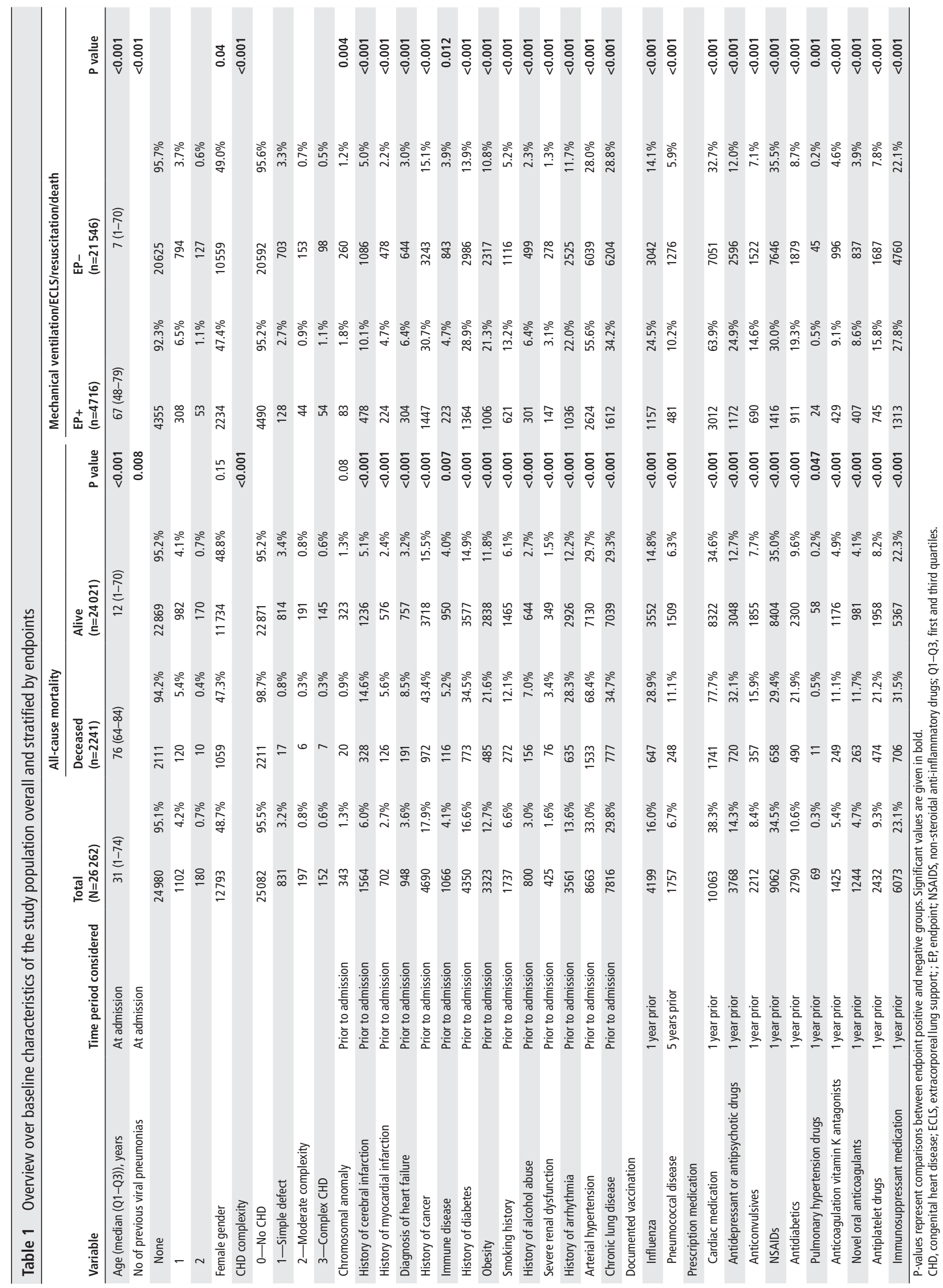




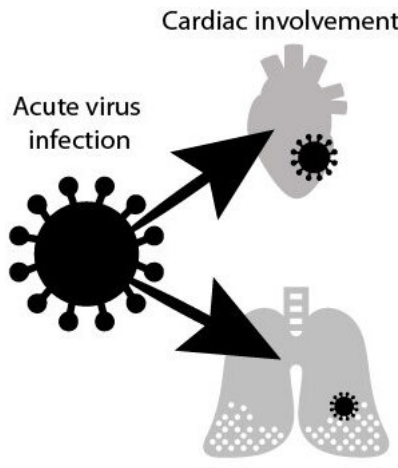

Viral pneumonia

Acute respiratory distress syndrome

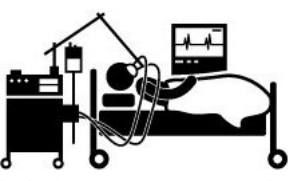

High risk of death or severe complications in patients with congenital heart disease
Figure 2A illustrates the relationship between age and event rates in patients with and without CHD, again illustrating that the former reach event rates observed in elderly patients without CHD ( $>60$ years of age) already from the onset of adulthood on.

Regarding the composite endpoint of need for mechanical ventilation/ECLS, resuscitation or death, event rates in patients with CHD were again consistently higher compared with patients without CHD: $18.0 \%$ vs $5.0 \%$ at age $0-9,23.4 \%$ vs $8.1 \%$ at age $10-19$ and $40.0 \%$ vs $27.6 \%$ at age $20-59$ years. Figure $2 \mathrm{~B}$ shows the event rate as a function of age for patients with and without CHD. In addition, it provides information on the relative risk ratio between various CHD age groups and matched patients without CHD. It shows that while CHD children have relatively lower absolute risks of complications, when compared with their age-matched non-CHD peers, their relative risk increase for death or severe acute complications is substantial, ranging between 3.5 and 6.3 .

On univariable and multivariable logistic regression analyses, CHD complexity, heart failure, a history of cardiac arrhythmias as well as a number of extracardiac comorbidities emerged as significant predictors of outcome. Table 2 illustrates the results of the multivariable logistic regression analysis in the entire study population. It shows that age, previous virus pneumonia episodes, male gender, complexity of cardiac defects, concomitant chromosomal anomalies, history of a cancer diagnosis, obesity, smoking and alcohol abuse as well as use of cardiac medications, antipsychotic drugs or anticonvulsives, antidiabetic medication and immunosuppressants represent multivariable predictors of adverse outcome. In contrast, vaccination against influenza was associated with lower event rates in this analysis, and a trend toward lower event rates in patients vaccinated against pneumococcal disease was seen. The results of the univariable analyses for the entire cohort and specifically for the CHD cohort are presented in the online supplemental material. Chronic lung disease was defined relatively broadly in the current analysis and this may account for the counterintuitive association between lung disease and outcome.

Even patients with simple cardiac defects had significantly increased risks of adverse outcome (OR 1.74 and 1.96 for death/ resuscitation/mechanical cardiac support and transplantation and mechanical ventilation/ECLS/death compared with noncongenital patients). However, when assessing specifically adult patients with CHD with simple defects who experienced events, we found that $87.5 \%-90 \%$ of patients had concomitant risk factors depending on endpoints considered. For example, $12 \%$ of the adult patients with CHD with simple defects who died had heart failure, 18\% had a history of cardiac arrhythmias, $41 \%$ required cardiac medication, $29 \%$ had a cancer diagnosis and $35 \%$ were on immunosuppressive drugs. In addition, the median age of the adult patients with simple defects who experienced events in the current analysis was 66 years. In fact, all patients with simple defects who experienced events or died below the age of 60 had additional cardiovascular risk factors or associated severe health conditions in our analysis.

\section{DISCUSSION}

Based on a large nationwide administrative dataset of in-hospital viral pneumonia cases in 24980 patients over a 13 -year period, our study provides novel mortality and morbidity estimates for affected patients with CHD. The analysis shows that patients with CHD had a worse outcome compared with their age-matched non-CHD peers, and mortality or morbidity rates in young or middle-aged adult patients with CHD were comparable to those observed in non-congenital individuals aged 60 years and above. Therefore, the current data suggest that patients with CHD with moderate and complex cardiac defects should be considered higher risk patients in the setting of severe viral infections and this information may help to guide medical management and health policy decisions in this vulnerable population. While in our analysis children with CHD exhibited lower 


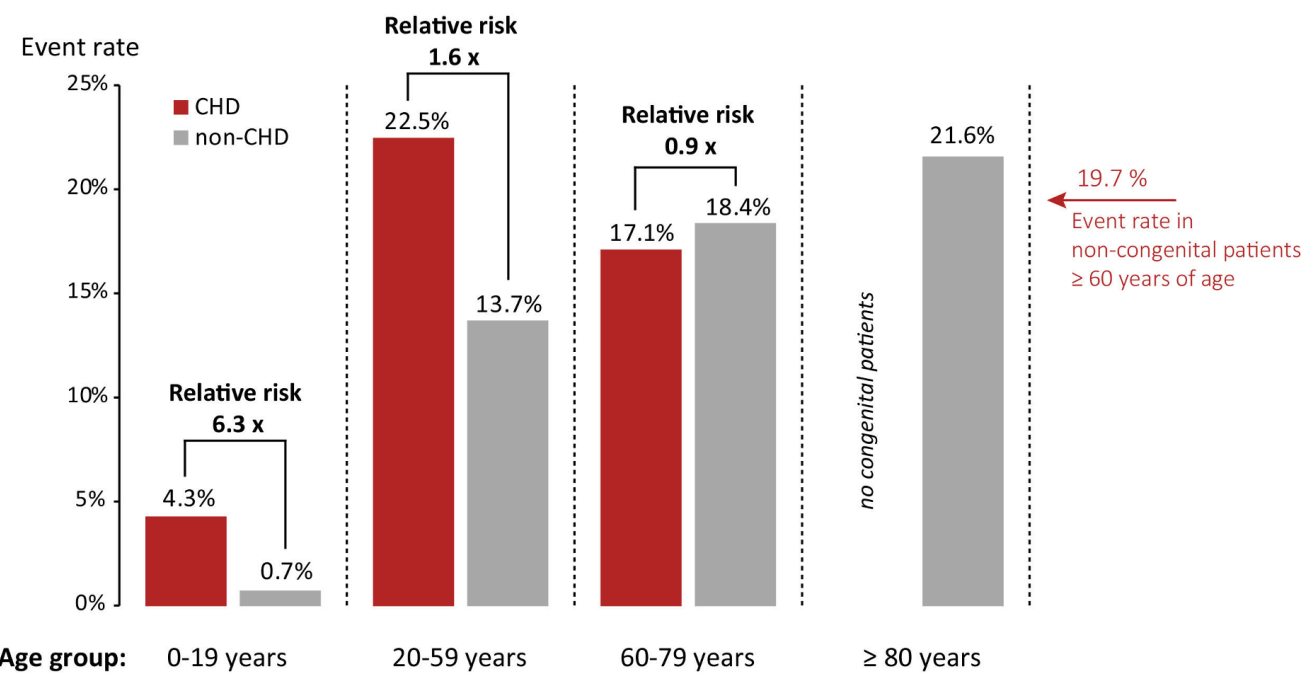

Figure 2 (A) Association between age and the composite endpoint of death, resuscitation, mechanical cardiac support (MCS) and transplantation in patients with congenital heart disease (CHD) and individuals without congenital heart defects showing that risk of severe complications increases early in adulthood in patients with CHD, reaching levels only seen in elderly non-congenital individuals. (B) In addition, a similar effect is seen for the composite endpoint of mechanical ventilation, extracorporeal lung support (ECLS). CPR, cardiopulmonaryresuscitation.

absolute mortality and complication rates overall, their relative risk of adverse outcomes was nevertheless 3.5 to 6 -fold higher compared with their non-congenital peers. As a consequence, even children with CHD could be regarded as higher-risk subjects based on the current analysis. We also identified comorbidities associated with higher risk of death or adverse outcome, including older age, male gender, complexity of the underlying heart defect, chromosomal anomalies, cancer, cardiac medication as well as immunosuppressive drugs.

While viral pneumonias primarily target the lungs, the disease is frequently accompanied by cardiovascular complications. ${ }^{9} 20$
Furthermore, it has been highlighted that patients with pre-existing cardiovascular conditions are not only more susceptible for viral infections but subsequently also exhibit worse clinical outcomes compared with healthy subjects. ${ }^{9}{ }^{20-24}$ Many patients with CHD also present with additional health issues including pulmonary disease, immune defects or multisystem disorders in addition to the underlying cardiac defect. ${ }^{48}$ As a consequence, these patients are prime candidates for complications and increased mortality when faced with severe viral pneumonia. The current SARS-CoV-2 pandemic has highlighted uncertainties associated with viral pneumonia in CHD cohorts, as this aspect has 


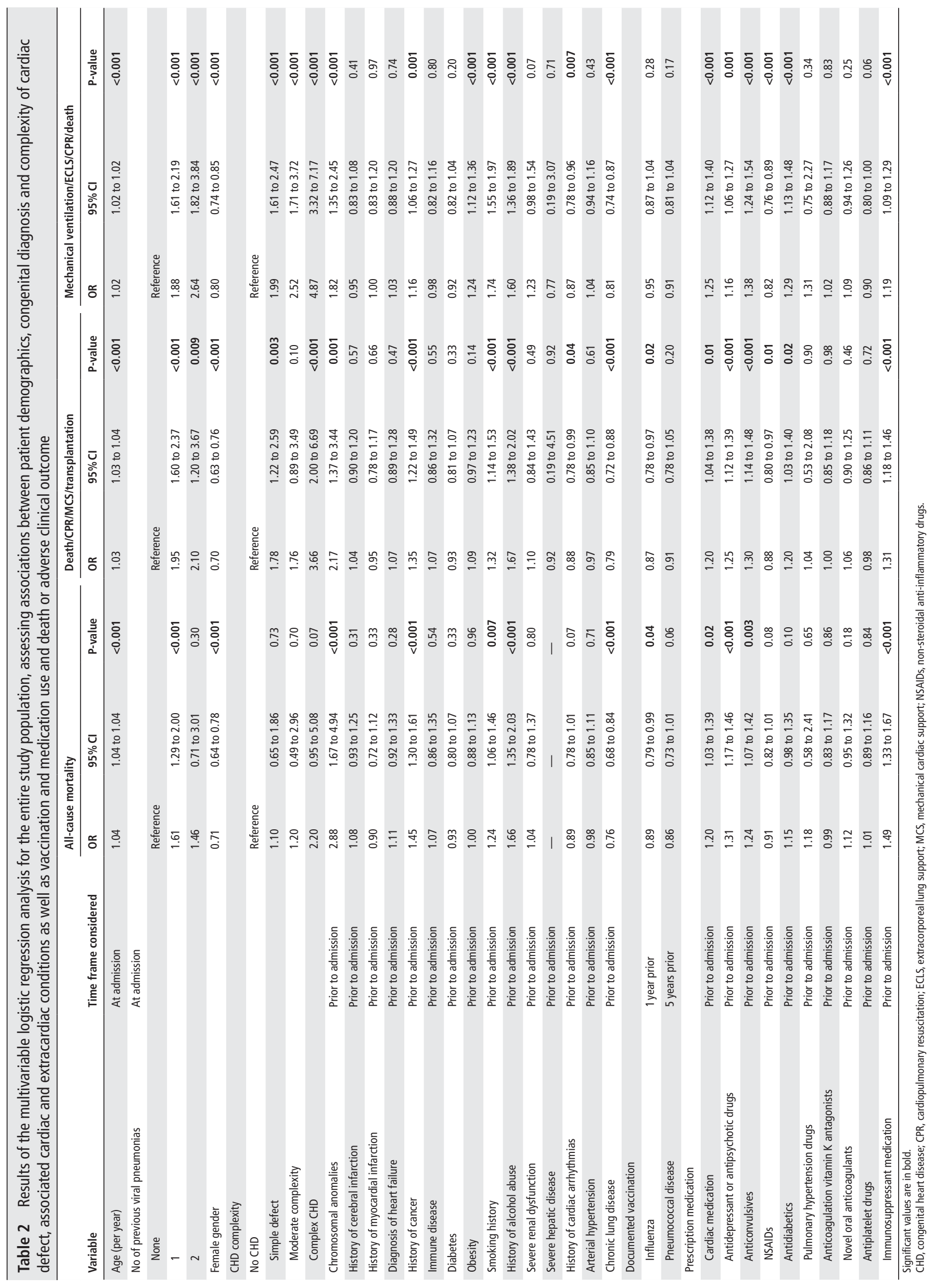


received much less attention traditionally compared with bacterial infections and endocarditis. Efforts are under way to collect prospective data on COVID-19 infection outcomes and provide recommendations specifically for patients with CHD. ${ }^{15}{ }^{25}$ These specific data are urgently required to guide front-line clinicians, politicians and patients. Our data may complement this information as it has been emphasised that the severity and outcome of coronavirus infections may-in part-be similar to other respiratory viruses, most notably influenza. ${ }^{12}$ Beyond shared pathogenic mechanisms, clinical manifestations and even radiological appearances, the pathophysiological responses between the conditions are relatively similar. ${ }^{9} 122627$ Therefore, these general information on outcome and complications of viral pneumonias in patients with CHD could at least serve as a basis for expert discussion and recommendations making. Also, the information presented here is relevant beyond COVID-19 as clinicians are commonly faced with viral pneumonias unrelated to SARS-CoV-2 infections and future epidemics of other viruses can be expected.

In our analysis, severity of the cardiac defect emerged as a risk factor for adverse outcome. Patients with complex underlying cardiac defects had the highest risk on multivariable logistic regression analysis (OR 4.8 compared with patients without CHD), followed by medium complexity patients (OR 2.5 compared with patients without CHD). Even patients with CHD with simple underlying cardiac defects had increased mortality and complication rates overall. However, as a group, adult patients with CHD with simple defects were on average older (median 66 years) than those with more complex lesions and approximately $90 \%$ of the event patient in the simple complexity group had additional risk factors, potentially explaining the higher risk. None of the adult patients with CHD with simple underlying cardiac lesions without additional cardiovascular risk factors or severe associated health issues were affected by need for ventilation/ECLS or death in our analysis. Therefore, risk of adverse outcome and death appears very low in adults with CHD of low complexity, aged $<60$ years and no additional risk factors.

\section{Management implications}

Accepting that patients with CHD should be regarded as higher risk individuals, infection prevention is of paramount importance. Consistent with current guideline recommendations, ${ }^{428}$ we found vaccination against influenza and possibly pneumococcal pneumonia to be associated with lower risk of complications and mortality in the current study, suggesting that vaccinations should be offered liberally to patients with CHD. Interestingly, non-steroidal anti-inflammatory drugs prescribed before hospitalisation were not found to be associated with worse outcome in our analysis and if anything seemed to be associated with less complications in this population. While specific antiviral treatments exist for influenza infections, ${ }^{29}$ only supportive therapy can be offered for most other viral infections. These measures include oxygen, antipyretic therapy and adequate therapy of the underlying cardiac condition as well as pre-existing or developing end-organ complications. ${ }^{15}$

\section{Strength of the current report}

To the best of our knowledge, this is the first large analysis of mortality and severe complication rates in patients with CHD suffering from severe viral pneumonia requiring hospitalisation. The current report highlights the high morbidity and mortality rate of patients with CHD with viral pneumonias requiring in-hospital treatment and has direct medical and health policy implication for the current pandemic and beyond, potentially providing a basis for a rational approach.

\section{Limitations}

Our analysis is focused on cases of viral pneumonia requiring hospitalisation. Therefore, it is biased toward more severe cases of virus infection. Thus, CFRs and morbidity data presented represents the in-hospital fatality/complication rates. The presented data overestimate the morbidity and mortality of disease in the community and should not be directly applied to ambulatory patients with viral pneumonia. We accept that this is a retrospective report based on administrative data, covering the pre-COVID-19 period. As such viral pneumonia studied here is due to different viruses, mostly influenza. Therefore, the results described here should not be directly extrapolated to SARS-CoV-2. Despite these important limitations, however, careful and critical consideration of experience with viral pneumonia in general may have implications for the management of emerging viruses and has also been advocated specifically for COVID-19. ${ }^{12}$

\section{CONCLUSIONS}

We present novel and timely data on the morbidity and mortality associated with viral pneumonia in patients with $\mathrm{CHD}$ requiring hospital admission. Our data illustrate the high morbidity and mortality of viral pneumonias in hospitalised patients with CHD, irrespective of age. Need for mechanical ventilation and risk of death in CHD increased early in life in patients with $\mathrm{CHD}$, reaching a level equivalent to non-CHD individuals $>60$ years of age. This may provide a rationale for classifying patients with CHD with moderately and highly complex defects as well as those with simple defects and associated risk factors as highrisk individuals and may have implications for current and future virus epidemics.

Key messages

What is already known on this subject?

- Limited data exist on the clinical outcome of patients with congenital heart disease (CHD) affected by severe viral pneumonia requiring in-hospital treatment.

\section{What might this study add?}

- Patients with CHD exhibit an increased risk of mortality and morbidity compared with non-congenital cohorts when affected by severe viral pneumonia requiring hospital admission. The morbidity and mortality risk of young and middle-aged patients with CHD is similar to that observed in patients without CHD aged $>60$ years of age. Age, CHD complexity, chromosomal anomalies, cardiac medication, use of immunosuppressants and absence of vaccination for influenza emerged as risk factors of adverse outcome.

\section{How might this impact on clinical practice?}

- The results of the current study should inform recommendations on risk stratification and management of patients with CHD with viral pneumonia, including pandemic situations.

Twitter Robert M Radke @dr_radke

Contributors G-PD and DE had full access to all the data in the study and takes responsibility for the integrity of the data and the accuracy of the data analysis. 
Concept and design: G-PD, HB and DE. Acquisition and statistical analysis: DE and JG. Drafting of the manuscript: G-PD and AEL. Critical revision of the manuscript for important intellectual content: RMR, SO, RS, FDTA, GK, UMB and UM. Obtained funding: G-PD and HB. Supervision: G-PD, HB and JG.

Funding The study is conducted within the framework of the OptAHF project (Optimizing the care in ACHD; GBA innovation fonds 2018). This study was supported by a research grant from provided by the EMAH Stiftung Karla VÖLLM, Krefeld, Germany.

Competing interests None declared.

Patient consent for publication Not required.

Provenance and peer review Not commissioned; externally peer reviewed.

Data availability statement No data are available. Data cannot be made publicly available as the analysis is based on the anonymised dataset of the BARMER Health Insurance Company.

Supplemental material This content has been supplied by the author(s). It has not been vetted by BMJ Publishing Group Limited (BMJ) and may not have been peer-reviewed. Any opinions or recommendations discussed are solely those of the author(s) and are not endorsed by BMJ. BMJ disclaims all liability and responsibility arising from any reliance placed on the content. Where the content includes any translated material, BMJ does not warrant the accuracy and reliability of the translations (including but not limited to local regulations, clinical guidelines, terminology, drug names and drug dosages), and is not responsible for any error and/or omissions arising from translation and adaptation or otherwise.

Open access This is an open access article distributed in accordance with the Creative Commons Attribution Non Commercial (CC BY-NC 4.0) license, which permits others to distribute, remix, adapt, build upon this work non-commercially, and license their derivative works on different terms, provided the original work is properly cited, appropriate credit is given, any changes made indicated, and the use is non-commercial. See: http://creativecommons.org/licenses/by-nc/4.0/.

\section{ORCID iDs}

Gerhard-Paul Diller http://orcid.org/0000-0003-3050-5248

Robert M Radke http://orcid.org/0000-0002-5055-2220

Fernando De Torres Alba http://orcid.org/0000-0002-1845-9704

\section{REFERENCES}

1 Khairy P, lonescu-Ittu R, Mackie AS, et al. Changing mortality in congenital heart disease. J Am Coll Cardiol 2010;56:1149-57.

2 Marelli AJ, lonescu-Ittu R, Mackie AS, et al. Lifetime prevalence of congenital heart disease in the general population from 2000 to 2010. Circulation 2014;130:749-56.

3 Moons P, Bovijn L, Budts W, et al. Temporal trends in survival to adulthood among patients born with congenital heart disease from 1970 to 1992 in Belgium. Circulation 2010;122:2264-72.

4 Baumgartner $\mathrm{H}$, Bonhoeffer $\mathrm{P}$, De Groot NMS, et al. ESC guidelines for the management of grown-up congenital heart disease (new version 2010). Eur Heart J 2010;31:2915-57.

5 Stout KK, Daniels CJ, Aboulhosn JA, et al. 2018 AHA/ACC guideline for the management of adults with congenital heart disease: a report of the American College of Cardiology/American heart association Task force on clinical practice guidelines. Circulation 2019;139:e698-800.

6 Diller G-P, Kempny A, Alonso-Gonzalez R, et al. Survival prospects and circumstances of death in contemporary adult congenital heart disease patients under follow-up at a large tertiary centre. Circulation 2015;132:2118-25.
7 Diller G-P, Baumgartner H. Endocarditis in adults with congenital heart disease: new answers-new questions. Eur Heart J 2017;38:2057-9.

8 Alonso-Gonzalez R, Borgia F, Diller G-P, et al. Abnormal lung function in adults with congenital heart disease: prevalence, relation to cardiac anatomy, and association with survival. Circulation 2013;127:882-90.

9 Zheng Y-Y, Ma Y-T, Zhang J-Y, et al. COVID-19 and the cardiovascular system. Nat Rev Cardiol 2020;17:259-60.

10 Bonow RO, Fonarow GC, O'Gara PT, et al. Association of coronavirus disease 2019 (COVID-19) with myocardial injury and mortality. JAMA Cardiol 2020;5:751-3.

11 Nguyen JL, Yang W, Ito K, et al. Seasonal influenza infections and cardiovascular disease mortality. JAMA Cardio/ 2016;1:274-81.

12 Vardeny O, Madjid M, Solomon SD. Applying the lessons of influenza to COVID-19 during a time of uncertainty. Circulation 2020;141:1667-9.

13 Simonsen L, Spreeuwenberg P, Lustig R, et al. Global mortality estimates for the 2009 influenza pandemic from the GLaMOR project: a modeling study. PLoS Med 2013:10:e1001558.

14 Centers for Disease Control and Prevention. Disease burden of influenza, 2020. Available: https://www.cdc.gov/flu/about/burden/index.html [Accessed $17 \mathrm{Apr}$ 2020].

15 Tan WM, Aboulhosn J. Coronavirus disease 2019 (COVID-19) and cardiovascular burden including congenital heart disease. International Journal of Cardiology. In Press 2020.

16 Fang Y, Zhang H, Xie J, et al. Sensitivity of chest CT for COVID-19: comparison to RTPCR. Radiology 2020;296:E115-7.

17 Liu PP, Blet A, Smyth D, et al. The science underlying COVID-19: implications for the cardiovascular system. Circulation 2020;142:68-78.

18 Hendren NS, Drazner MH, Bozkurt B, et al. Description and proposed management of the acute COVID-19 cardiovascular syndrome. Circulation 2020;141:1903-14.

19 Freisinger E, Koeppe J, Gerss J, et al. Mortality after use of paclitaxel-based devices in peripheral arteries: a real-world safety analysis. Eur Heart J. In Press 2019;2019. doi:10.1093/eurheartj/ehz698. [Epub ahead of print: 08 Oct 2019].

20 Tan W, Aboulhosn J. The cardiovascular burden of coronavirus disease 2019 (COVID-19) with a focus on congenital heart disease. Int J Cardiol. In Press 2020;309:70-7.

21 Lippi G, Lavie CJ, Sanchis-Gomar F. Cardiac troponin I in patients with coronavirus disease 2019 (COVID-19): evidence from a meta-analysis. Prog Cardiovasc Dis 2020;63:390-1.

22 Shi S, Qin M, Shen B, et al. Association of cardiac injury with mortality in hospitalized patients with COVID-19 in Wuhan, China. JAMA Cardiol 2020;5:802-10.

23 Ukimura A, Satomi H, Ooi Y, et al. Myocarditis associated with influenza A H1N1pdm2009. Influenza Res Treat 2012;2012:351979.

24 Yu C-M, Wong RS-M, Wu EB, et al. Cardiovascular complications of severe acute respiratory syndrome. Postgrad Med J 2006;82:140-4.

25 Radke RM, Frenzel T, Baumgartner $\mathrm{H}$, et al. Adult congenital heart disease and the COVID-19 pandemic. Heart 2020;106:1302-9.

26 Kim EA, Lee KS, Primack SL, et al. Viral pneumonias in adults: radiologic and pathologic findings. Radiographics 2002;22 Spec No:S137-49.

27 Korteweg C, Gu J. Pathology, molecular biology, and pathogenesis of avian influenza A (H5N1) infection in humans. Am J Pathol 2008;172:1155-70.

28 Lui GK, Saidi A, Bhatt AB, et al. Diagnosis and management of noncardiac complications in adults with congenital heart disease: a scientific statement from the American heart association. Circulation 2017;136:e348-92.

29 Nicholson KG, Aoki FY, Osterhaus AD, et al. Efficacy and safety of oseltamivir in treatment of acute influenza: a randomised controlled trial. neuraminidase inhibitor flu treatment investigator group. Lancet 2000;355:1845-50. 